
DES ALTEN ORIENTS

\begin{abstract}
AKADEMIE
DER WISSENSCHAFTEN

DER DDR

ZENTRALINSTITUT

FÜR ALTE GESCHICHTE

UND ARCHÄOLOGIE
\end{abstract}

\title{
RALF-BERNHARD WARTKE
}

\section{Toprakkale}

Untersuchungen zu den Metallobjekten im Vorderasiatischen Museum zu Berlin

Mit 37 Abbildungen im Text und 43 Tafeln 
ISBN 3-05-000921-7

ISSN 0080-6994

Redaktion: Marie-Luise Dunkelmann

Erschienen im Akademie-Verlag Berlin, Leipziger Str. 3-4, Berlin, DDR - 1086

(C) Akademie-Verlag Berlin 1990

Printed in the German Democratic Republic

Herstellung: VEB Druckerei „Gottfried Wilhelm Leibniz“, 4450 Gräfenhainichen · 7288

Lektorin: Heidi Lässer

LSV 0225

Bestellnummer: $7550712(2146 / 22)$ 